\title{
Electrochemical Properties of Acetylene Black/Multi-walled Carbon Nanotube Cathodes for Lithium Thionyl Chloride Batteries at High Discharge Currents
}

\author{
Hee-Youb Song ${ }^{1}$, Moon-Hyung Jung ${ }^{2}$, and Soon-Ki Jeong ${ }^{3, *}$ \\ ${ }^{1}$ VIZTRO MILTECH., Cheonan, Chungnam, 31214, Korea \\ ${ }^{2}$ VITZROCELL Co., Ltd., 70, Indeoseupakeu, Hapdeok, Dangjin, Chungnam 31816, Korea \\ ${ }^{3}$ Department of Energy Systems, Soonchunhyang University, Asan, Chungnam, 31538, Korea
}

\begin{abstract}
Lithium thionyl chloride $\left(\mathrm{Li} / \mathrm{SOCl}_{2}\right)$ batteries exhibit the highest energy densities seen in commercially available primary batteries because of their high operating voltages and discharge capacities. They are widely used in various extreme environments; however, they show signs of degradation at high discharge currents. The discharge performance of $\mathrm{Li} / \mathrm{SOCl}_{2}$ is considered to be greatly dependent on the carbon materials used in the cathode. Therefore, suitable carbon materials must be chosen to improve discharge performances. In this work, we investigated the discharge properties of $\mathrm{Li} / \mathrm{SOCl}_{2}$ batteries in which the cathodes contained various ratios of acetylene black $(\mathrm{AB})$ and multi-walled carbon nanotubes (MWCNTs) at high discharge currents. It was confirmed that the MWCNTs were effectively dispersed in the mixed AB/MWCNT cathodes. Moreover, the discharge capacity and operating voltage improved at high discharge currents in these mixed cathodes when compared with pure $\mathrm{AB}$ cathodes. It was found that the mesopores present in the cathodes have a strong impact on the discharge capacity, while the macropores present on the cathode surface influence the discharge properties at high discharge rates in $\mathrm{Li} / \mathrm{SOCl}_{2}$ batteries. These results indicate that the ratio of mesopores and macropores in the cathode is key to improving the discharge performance of $\mathrm{Li} / \mathrm{SOCl}_{2}$ batteries, as is the dispersion of the MWCNTs.
\end{abstract}

Keywords : Lithium Thionyl Chloride, Multi-Wall Carbon Nanotube, Acetylene Black, Lithium Primary Batteries

Received : 9 July 2020, Accepted : 13 August 2020

\section{Introduction}

Lithium thionyl chloride $\left(\mathrm{Li} / \mathrm{SOCl}_{2}\right)$ primary batteries have been widely used as power sources in extreme environments because of their high operating voltage $(3.6 \mathrm{~V})$, wide operating temperature range $\left(-55-85^{\circ} \mathrm{C}\right)$, and high discharge capacity $(0.8$ $\mathrm{Ah}, \mathrm{AA}$ size, wound type) [1-4]. In general, $\mathrm{Li} / \mathrm{SOCl}_{2}$ batteries consist of a lithium metal anode, a carbonbased cathode, and $\mathrm{SOCl}_{2}$ containing a lithium salt as the electrolyte solution. During the discharge process, the lithium metal is oxidized and the $\mathrm{SOCl}_{2}$ electrolyte solution is reduced to produce sulfur, sulfur dioxide, and lithium chloride. The carbon cathode

*E-mail address: hamin611@sch.ac.kr

DOI: https://doi.org/10.33961/jecst.2020.01179

This is an open-access article distributed under the terms of the Creative Commons Attribution Non-Commercial License (http://creativecommons.org/licenses/by-nc/4.0) which permits unrestricted non-commercial use, distribution, and reproduction in any medium, provided the original work is properly cited. acts as a catalytic surface for the reduction of the $\mathrm{SOCl}_{2}[1-3]$, and the surface structure of the carbon cathode is of particular importance due to the formation of insoluble reaction products such as lithium chloride on the carbon surface [5-11]. Lithium chloride exhibits a low electrical conductivity, thereby hindering the reduction of $\mathrm{SOCl}_{2}$, and so the presence of lithium chloride in the pores of the carbon cathode can make the diffusion of $\mathrm{SOCl}_{2}$ during the discharge process more difficult. These issues must therefore be addressed to allow for the broader application of $\mathrm{Li} /$ $\mathrm{SOCl}_{2}$ batteries.

The discharge performances of $\mathrm{Li} / \mathrm{SOCl}_{2}$ batteries are greatly dependent on the physicochemical properties of the carbon cathode materials employed. For example, carbon nanotubes (CNTs) could improve the discharge performance at high discharge currents due to their high electrical conductivity and high specific surface area. As such, the catalytic effects of 
CNTs in $\mathrm{Li} / \mathrm{SOCl}_{2}$ batteries have been widely investigated [12-19]. However, the impact of their structure on the discharge performance of $\mathrm{Li} / \mathrm{SOCl}_{2}$ batteries is not yet clearly understood. Additionally, their nanostructures allow the facile formation of aggregates, which may cause a deterioration in the battery performance. Therefore, technologies aimed at the dispersion of CNTs in the cathodes are key to improving the discharge performances of $\mathrm{Li} / \mathrm{SOCl}_{2}$ batteries. Commercially, mixtures of acetylene black $(\mathrm{AB})$ and ketjen black have been widely used as a cathode material in $\mathrm{Li} / \mathrm{SOCl}_{2}$ batteries. However, the discharge performances of such systems at high discharge rates are generally poor under extreme environments. To address this issue, we wished to consider a new composite electrode, namely $\mathrm{AB} /$ multi-walled carbon nanotube (MWCNT), as the battery cathode material, with the aims of improving the discharge performance at high discharge rates. Thus, we herein report our investigation into the effect of $\mathrm{AB}$ addition on the effective dispersion of MWCNTs in cathodes, and the impact of mixing $\mathrm{AB}$ and MWCNTs on the discharge performance of $\mathrm{Li} / \mathrm{SOCl}_{2}$ batteries by investigating the discharge properties of $\mathrm{AB} / \mathrm{MWCNT}$ mixed cathodes at discharge currents of 150 and $600 \mathrm{~mA}$. In addition, the correlation between the discharge properties and the physical properties of AB/MWCNT mixed cathodes are discussed.

\section{Experimental}

\subsection{Preparation of cathode materials}

Cathode materials were prepared using AB (Denka black, Denka Co. Ltd., Tokyo, Japan) and MWCNTs (LUCAN BT1003M, LG Chem. Seoul, Korea) with $\mathrm{AB}: \mathrm{MWCNT}$ weight ratios of $0: 10,3: 7,5: 5,7: 3$, and 10:0. Isopropyl alcohol (99.7\%, Sigma-Aldrich Co., St. Louis, USA) was added to the cathode materials and these were stirred for $10 \mathrm{~min}$. Subsequently, pastes were prepared by mixing the cathode materials and polytetrafluoroethylene (POLYFLON PTFE DSeries, DAIKIN Industries Ltd., Osaka, Japan) in a 10:1 weight ratio and stirring for $5 \mathrm{~min}$. Expanded nickel mesh (VITZRO CELL Co. Ltd. Chungnam, Korea) was used as a current collector, and the pastes were pressed onto the mesh by roll-pressing. The cathode materials were loaded on the current collector at a loading of $\sim 0.6 \mathrm{~g}$. To maintain a constant mass loading, the thickness of the cathode was fixed at $0.7 \mathrm{~mm}$, and the cathodes were dried at $120^{\circ} \mathrm{C}$ for $4 \mathrm{~h}$ under vacuum.

\subsection{Materials characterization}

Scanning electron microscopy (SEM) was carried out to investigate the morphologies of the $\mathrm{AB} /$ MWCNT mixed cathodes (S-4800, Hitachi Ltd., Tokyo, Japan). The specific surface area and pore size distribution were investigated by nitrogen adsorption-desorption isotherm measurements (ASAP 2420, Micromeritics Instrument Corp., Norcross, USA).

\subsection{Electrochemical measurements}

Electrochemical tests were performed using commercial wound-type batteries (AA size), as shown in Fig. 1. Lithium metal (VITZRO CELL Co. Ltd. Chungnam, Korea) was used as the anode and the $\mathrm{AB} / \mathrm{MWCNT}$ mixed materials were used as the cathode. In addition, $1.25 \mathrm{M} \mathrm{LiAlCl}_{4}(98 \%$, BASF SE, Ludwigshafen, Germany) $/ \mathrm{SOCl}_{2}(99.6 \%$, LANXESS, Cologne, Germany) was selected as the electrolyte solution. The discharge tests were performed between the open circuit voltage (OCV) and $2.0 \mathrm{~V}$ at both 150 and $600 \mathrm{~mA}$ (Series 3600, MACCOR Inc., Oklahoma, USA). Nyquist plots of the $\mathrm{Li} / \mathrm{SOCl}_{2}$ batteries were obtained before discharge testing at $\mathrm{OCV}$ by electrochemical impedance spectroscopy (ZIVE MP5, WanATech Co. Ltd., Seoul, Korea). The frequency range was $100 \mathrm{kHz}-10 \mathrm{mHz}$ with an alternating amplitude of $10 \mathrm{mV}$.

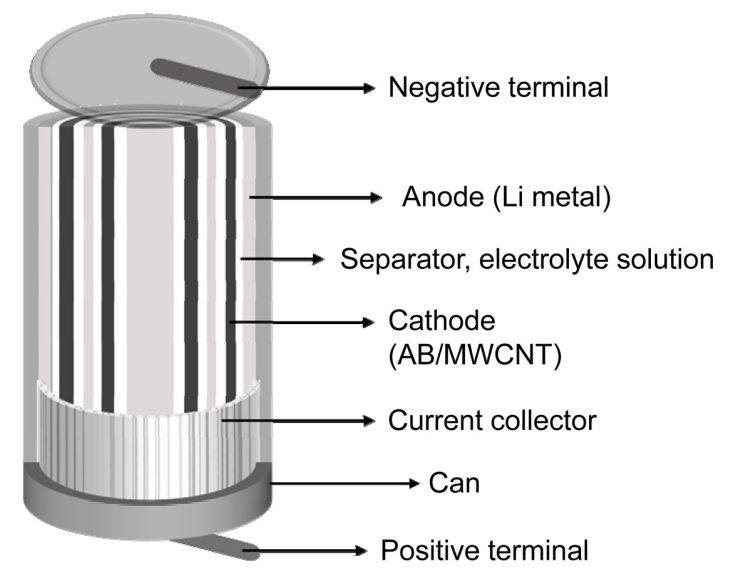

Fig. 1. Schematic representation of the wound-type Li/ $\mathrm{SOCl}_{2}$ battery. 

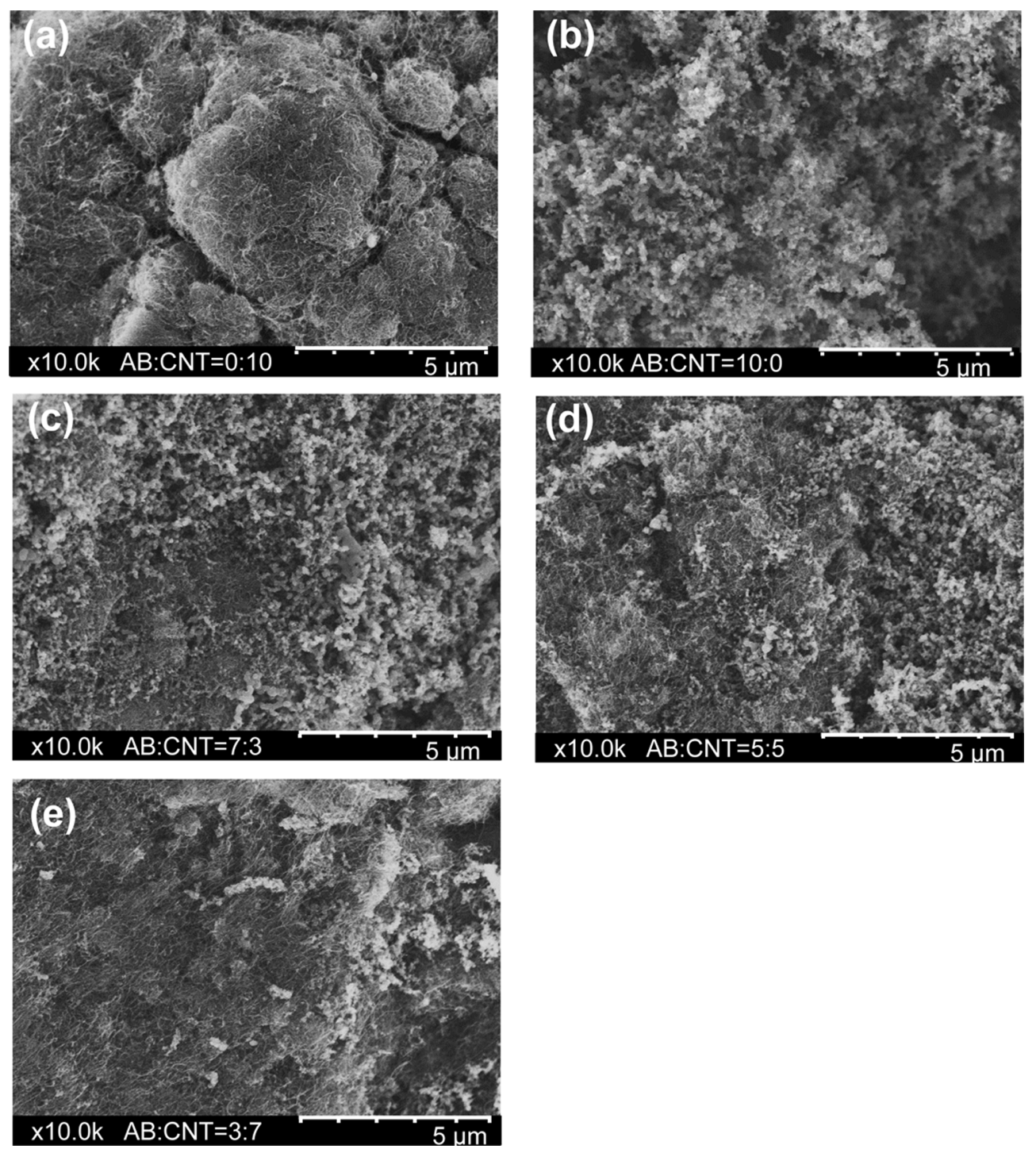

Fig. 2. SEM images of the $A B$ and MWCNT mixed cathodes, with AB:MWCNT ratios of (a) 0:10, (b) 10:0, (c) 5:5, (d) $7: 3$, and (e) $3: 7$.

\section{Results and Discussion}

3.1 Physical properties of the AB/MWCNT cathodes

Fig. 2 shows SEM images of the cathodes prepared using various $\mathrm{AB}: \mathrm{MWCNT}$ ratios. In the pure MWCNT cathode, mostly aggregated linear structures were observed, while spherical structures were confirmed in the pure $A B$ cathode (Figs. 2a and 2b). In the case of AB:MWCNT (7:3), AB was dominant on the cathode surface (Fig. 2c), while increasing amounts of MWCNTs were observed on the cathode surface as the MWCNT content was increased (Figs. $2 \mathrm{~d}$ and 2e). Moreover, MWCNT aggregates were not confirmed in $\mathrm{AB} / \mathrm{MWCNT}$ mixed cathodes, indicating that MWCNTs were effectively dispersed by the addition of $\mathrm{AB}$. These results revealed that the morphology of carbon cathodes is largely dependent on the ratio of $\mathrm{AB}$ to MWCNTs.

The adsorption and desorption isotherms of the AB/MWCNT mixed cathodes are shown in Fig. 3. 
(a)

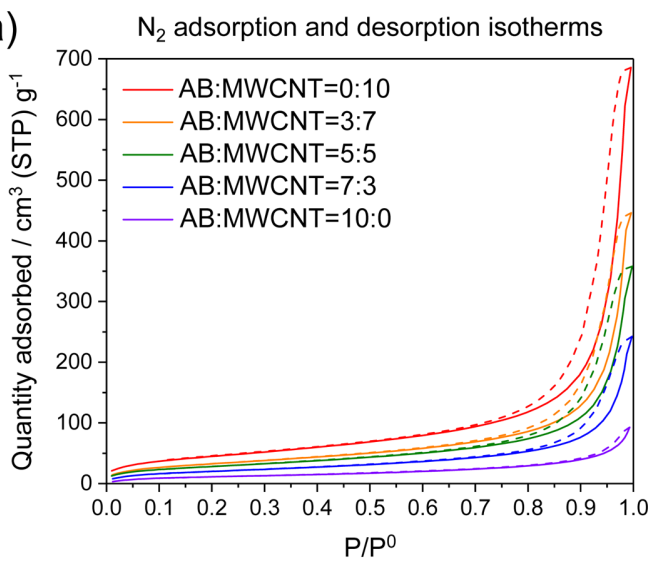

(b)

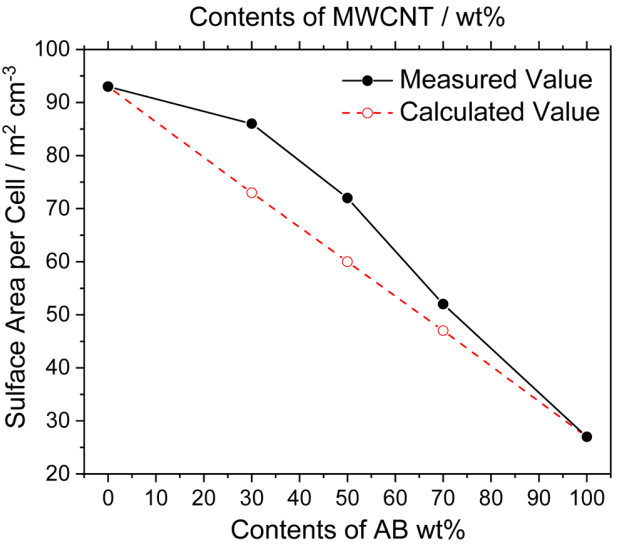

(c)

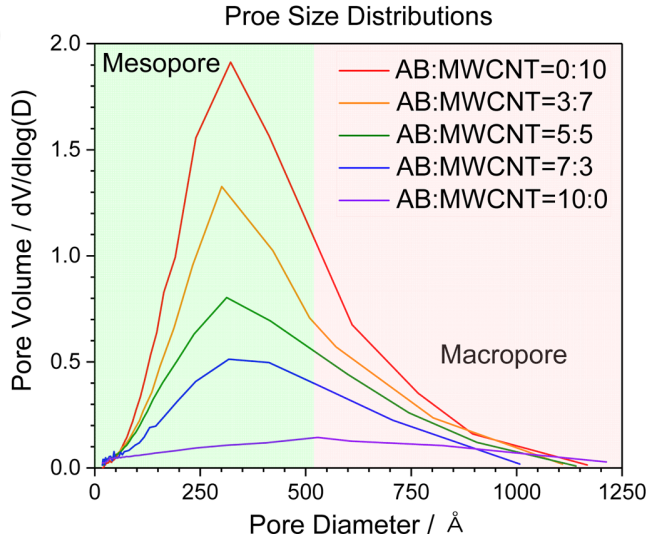

Fig. 3. Nitrogen adsorption/desorption isotherms and pore size distributions of the $A B$ and MWCNT mixed cathodes.

Table 1. Specific surface areas and specific pore volumes of the AB and MWCNT mixed cathodes

\begin{tabular}{ccccc}
\hline \hline \multirow{2}{*}{$\begin{array}{c}\text { Contents of electrodes } \\
\text { (AB:MWCNT) }\end{array}$} & $\begin{array}{c}\text { Specific surface area } \\
\left(\mathrm{m}^{2} \mathrm{~g}^{-1}\right)\end{array}$ & Mesopore $(\%)$ & Macropore $(\%)$ & ${\text { Total }\left(\mathrm{cm}^{3} \mathrm{~g}^{-1}\right)}^{$\cline { 3 - 4 }$}$ \\
\cline { 3 - 5 } & 166 & 78.2 & 21.8 & 1.20 \\
$3: 1$ & 122 & 76.3 & 23.7 & 0.87 \\
$5: 5$ & 114 & 75.4 & 24.6 & 0.56 \\
$7: 3$ & 76 & 71.0 & 29.0 & 0.41 \\
$10: 0$ & 43 & 63.5 & 36.5 & 0.15 \\
\hline
\end{tabular}

The isotherms are type IV, and the hysteresis indicates the existence of mesoporous structures within the cathodes (Fig. 3a) [14]. The largest specific surface area was observed for the pure MWCNT electrode, which was attributed to the smaller diameters of the MWCNTs, as well as their linear shape compared to that of $\mathrm{AB}$. The average diameter and the average length of the MWCNTs were estimated to be $13 \mathrm{~nm}$ and $12 \mu \mathrm{m}$, respectively, and the average diameter of $\mathrm{AB}$ was estimated to be $36 \mathrm{~nm}$. The calculated values (Fig. 3b) were obtained by interpolation between the values of MWCNTs and AB. It was confirmed that the measured specific surface areas of $\mathrm{AB} / \mathrm{MWCNT}$ mixed cathodes were larger than the 
calculated values, indicating that the cavities between the MWCNT particles were filled with AB particles. Indeed, large numbers of cavities were observed in the MWCNT aggregates, as shown in Fig. 2(a). Fig. 3 (c) shows the pore size distributions of the $\mathrm{AB} /$ MWCNT mixed cathodes, and detailed specific surface areas and specific pore volumes are shown in Table 1. Micropores with diameters of less than $2 \mathrm{~nm}$ were negligible in all electrodes, making up only $0.1 \%$, while mesopores with diameters of $2-50 \mathrm{~nm}$ were dominant $(>63.5 \%)$, with a low amount of macropores being detected in all cases, although this increased upon increasing the $\mathrm{AB}$ content. This result indicates that the ratio of $\mathrm{AB}$ to MWCNTs in mixed cathodes has a significant impact on the porosity of the system, which might be expected to alter the discharge properties of $\mathrm{Li} / \mathrm{SOCl}_{2}$ batteries constructed using these electrodes.

\subsection{Electrochemical properties of the AB/MWCNT cathodes in $\mathrm{Li} / \mathrm{SOCl}_{\mathbf{2}}$ batteries}

Fig. 4 shows the discharge properties of the Li/ $\mathrm{SOCl}_{2}$ batteries prepared using cathodes containing various $\mathrm{AB}$ :MWCNT ratios at 150 and $600 \mathrm{~mA}$. At a discharge current of $150 \mathrm{~mA}$, both the discharge time and the operating voltage increased in the MWCNTcontaining cathodes compared with the pure $\mathrm{AB}$ cathode (Fig. 4a). For the AB/MWCNT mixed cathodes, the specific surface area increased upon increasing the MWCNT content (see above), which provided larger reaction sites for the formation of $\mathrm{LiCl}$. This result indicates that the discharge capacities of these $\mathrm{Li} / \mathrm{SOCl}_{2}$ batteries at relatively low discharge currents are affected by the specific surface area. However, different discharge properties were confirmed for the AB/MWCNT mixed cathodes at a discharge current of $600 \mathrm{~mA}$ (Fig. 4b). More specifically, discharge times were longer for the cathodes containing MWCNTs compared to those observed for the pure $\mathrm{AB}$ cathode. In addition, the discharge times gradually increased upon increasing the $\mathrm{AB}$ content when using the mixed $\mathrm{AB} / \mathrm{MWCNT}$ cathodes. This result implied that the discharge performance at a high discharge current was enhanced for MWCNT cathodes upon the addition of AB. In MWCNT cathodes without $A B$, the majority of the MWCNTs were aggregated, leading to a deterioration in the discharge performance of $\mathrm{Li} / \mathrm{SOCl}_{2}$ at a high discharge current of $600 \mathrm{~mA}$. In addition, $\mathrm{LiCl}$ (a)

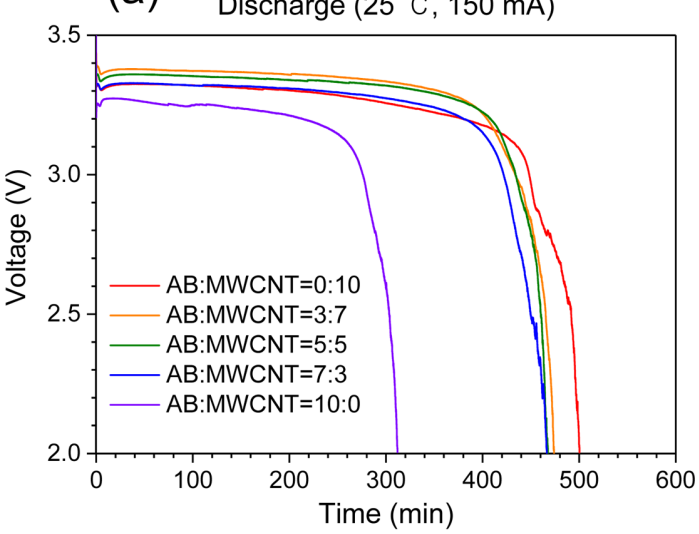

(b)

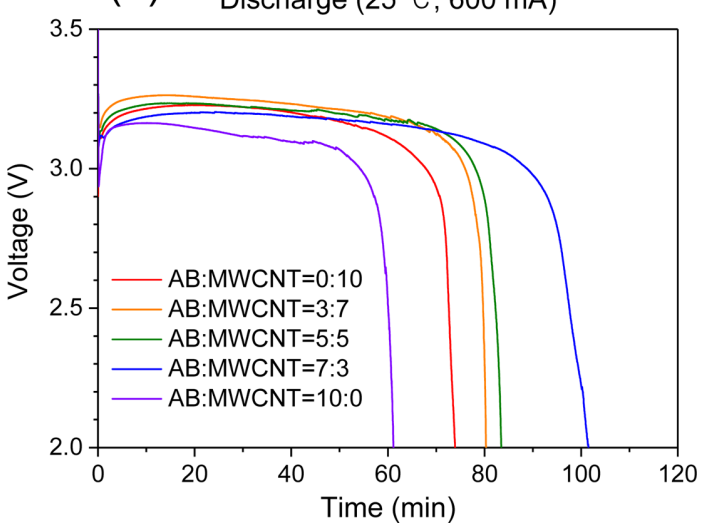

Fig. 4. Discharge curves of $\mathrm{Li} / \mathrm{SOCl}_{2}$ batteries prepared using cathodes containing various $\mathrm{AB}: \mathrm{MWCNT}$ ratios at discharge currents of (a) 150 and (b) $600 \mathrm{~mA}$.

forms more rapidly on the cathode surface at high discharge currents, causing pore closure in a significant number of mesopores. The longest discharge time was observed when using a mixed $A B /$ MWCNT cathode with a ratio of 7:3. In this case, $A B$ was predominant on the surface, leading to a greater number of macropores than in the case of the $\mathrm{AB} /$ MWCNT cathodes with ratios of 5:5 and 3:7 (Fig. 3 and Table 1). It was therefore apparent that the increased number of macropores on the cathode surface improved the discharge performance at high discharge rates. However, the AB cathode exhibited the lowest discharge capacity despite having the highest ratio of macropores on its surface, indicating that macropores alone do not determine the discharge capacity of $\mathrm{Li} / \mathrm{SOCl}_{2}$. Instead, they assist the diffu- 
(a)

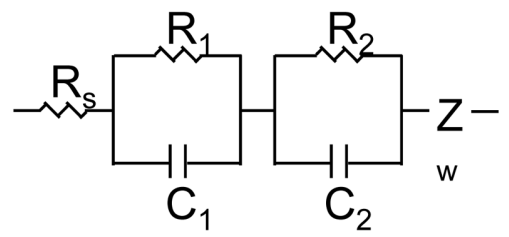

(b)

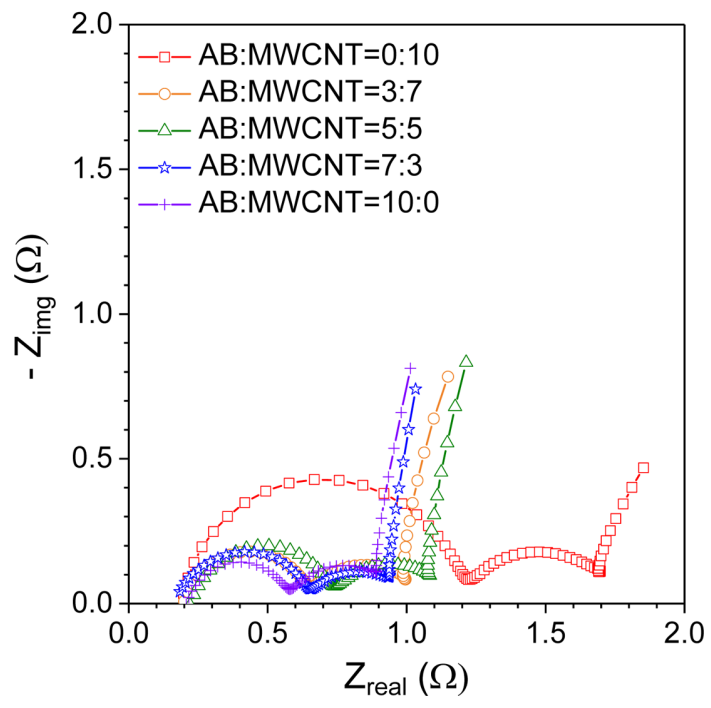

Fig. 5. (a) Equivalent circuit, and (b) Nyquist plots prior to discharge for the $\mathrm{Li} / \mathrm{SOCl}_{2}$ batteries in which the cathodes contained various AB:MWCNT ratios.

sion of $\mathrm{SOCl}_{2}$ toward the carbon cathode during the discharge process. It can therefore be considered that the discharge properties at a high discharge current of 600 $\mathrm{mA}$ are dependent on the number of macropores on the cathode surface, as well as the dispersion of MWCNTs.

Therefore, the ratio of mesopores to macropores in the cathode is important in the context of attempting to improve the discharge performance of $\mathrm{Li} / \mathrm{SOCl}_{2}$ at high discharge currents. Since the surface resistance of the carbon cathode is considered to have an influence on the discharge properties of $\mathrm{Li} / \mathrm{SOCl}_{2}$ batteries at high discharge currents, electrochemical impedance spectroscopy was carried out on the $\mathrm{AB} /$ MWCNT cathodes. The equivalent circuit and Nyquist plots of $\mathrm{Li} / \mathrm{SOCl}_{2}$ batteries with cathodes containing different ratios of $\mathrm{AB} / \mathrm{MWCNT}$ before discharge are shown in Fig. 5. The Nyquist plots shows two semicircles and one slope, whereby $R_{s}$,
$R_{1}, R_{2}, C_{1}, C_{2}$, and $Z_{w}$ (Fig. 5a) represent the solution resistance, surface film resistance, charge transfer resistance, surface film capacitance, double layer capacitance, and diffusion resistance, respectively [20-22]. The surface film resistance of the pure MWCNT cathode in the high frequency region was significantly larger than those seen for the mixed $\mathrm{AB} /$ MWCNT cathodes. This was a result of the high percentage of mesopores in the pure MWCNT cathode, as well as increased MWCNT aggregation. In addition, the surface film resistance decreased as the ratio of MWCNTs in the mixed AB/MWCNT cathodes decreased. Based on the above SEM images and pore size distributions, it appeared that an increased $A B$ content resulted in the generation of numerous macropores on the cathode surface. Moreover, the MWCNTs are more effectively dispersed in the mixed AB/MWCNT cathodes, and so the surface film resistance in the cathode was reduced upon the addition of $\mathrm{AB}$ to the MWCNTs. The slopes shown in the figure correspond to the Warburg impedance element, and are attributed to diffusion resistance in the cathodes. The diffusion coefficients were calculated using the following equation [23,24]:

$$
D=\frac{R^{2} T^{2}}{2 A^{2} n^{4} F^{4} C^{2} \sigma_{w}^{2}}
$$

where $\mathrm{D}, \mathrm{R}, \mathrm{T}, \mathrm{A}, \mathrm{n}, \mathrm{F}, \mathrm{C}$, and $\sigma_{\mathrm{w}}$ represent the diffusion coefficient, gas coefficient, absolute temperature, electrode area, number of electrons per molecule, Faraday's constant, molar concentration of lithium ions, and Warburg coefficient, respectively. The diffusion coefficients were estimated to be $7.10 \times 10^{-10}, 1.74 \times 10^{-9}, 2.08 \times 10^{-9}$, $4.00 \times 10^{-9}$, and $2.45 \times 10^{-9} \mathrm{~cm}^{2} \mathrm{~s}^{-1}$ for AB:MWCNT $=$ $0: 10,3: 7,5: 5,7: 3$, and 10:0, respectively. In the mixed AB/MWCNT cathodes, the diffusion coefficient increased upon decreasing the MWCNT content, which indicates that diffusion was faster in the mixed cathodes than in the pure MWCNT cathode. This result supports the discharge properties observed at a high discharge current of $600 \mathrm{~mA}$.

Based on these results, it therefore appears that the surface condition of carbon cathodes has an influence on the discharge performance of $\mathrm{Li} / \mathrm{SOCl}_{2}$ at high discharge currents. However, it alone does not determine the discharge capacity, as indicated by the short discharge time in the AB cathode. These results suggest that surface conditions should be controlled to give a relatively high macropore content to improve the rate performance. 
Moreover, the discharge capacity is determined by the mesopore ratio in the bulk of the carbon cathode.

\section{Conclusions}

We herein reported our investigation into the discharge properties of $\mathrm{Li} / \mathrm{SOCl}_{2}$ batteries in which the cathodes contained various ratios of $\mathrm{AB}$ and MWCNTs. For the purpose of this study, discharge currents of 150 and $600 \mathrm{~mA}$ were employed. SEM observations confirmed that the morphologies were significantly different in mixed AB/MWCNT cathodes. In addition, in the pure MWCNT cathodes, the majority of MWCNTs were aggregated, while they were effectively dispersed in mixed the AB/MWCNT cathodes, which resulted in a decrease in the surface resistance. Furthermore, pore size distribution analysis showed an increase in the mesopore ratio in the cathodes as the MWCNT content was increased. Moreover, the discharge times of $\mathrm{Li} / \mathrm{SOCl}_{2}$ batteries increased upon increasing the MWCNT content in mixed AB/MWCNT cathodes at a discharge current of $150 \mathrm{~mA}$ due to the greater specific surface area and larger number of mesopores. However, the discharge time decreased upon increasing the MWCNT content in the mixed AB/MWCNT cathodes at a discharge current of $600 \mathrm{~mA}$. In this case, $\mathrm{AB}$ was dominant on the surface of the cathodes, leading to the formation of numerous macropores on the surface. This result indicates that the discharge capacity is affected by the ratio of mesopores in the carbon cathodes. The macropores present on the surfaces of the carbon cathodes also influence the discharge performance of $\mathrm{Li} / \mathrm{SOCl}_{2}$ at high discharge currents. Thus, from our results, it is apparent that the discharge performance of $\mathrm{Li} / \mathrm{SOCl}_{2}$ batteries can be improved by using mixed $\mathrm{AB} / \mathrm{MWCNT}$ cathodes. In future work, the interfacial reactions that occur between the $\mathrm{SOCl}_{2}$ electrolyte and the cathode during the discharge process should be clarified to allow for commercialization.

\section{Acknowledgement}

This work was supported by the Korea Institute of Energy Technology Evaluation and Planning (KETEP) and the Ministry of Trade, Industry \& Energy (MOTIE) of the Republic of Korea (No. 20184030202130). This work also received support from the Soonchunhyang University Research Fund.

\section{References}

[1] A.J. Hills and N.A. Hampson, J. Power Sources, 1988, 24(4), 253-271.

[2] T. Placke, R. Kloepsch, S. Duhnen, and M. Winter, $J$. Solid State Electrochem., 2017, 21(7), 1939-1964.

[3] J.J. Auborn, K.W. French, S.I. Sheldon, I. Lieberman, Shah V.K., Heller A., J. Electrochem. Soc., 1973, 120(12), 1613.

[4] B.J. Carter, R.M. Williams, F.D. Tsay, A. Rodriguez, S. Kim, M.M. Evans, and H. Frank, J. Electrochem. Soc., 1985, 132(2), 525.

[5] A.N. Dey, Electrochim. Acta, 1976, 21(11), 855-860.

[6] V.S. Bagotzky, V.E. Kazarinov, Y.M. Volfkovich, L.S. Kanevsky, and L.A. Beketayeva, J. Power Sources, 1989, 26(3-4), 427-433.

[7] Y. Zhang and C.S. Cha, Electrochim. Acta, 1993, 38(6), 827-834.

[8] M. Jakic, M. Kovac, M. Gaberscek, and S. Pejovnik, Electrochim. Acta, 1995, 40(17), 2723-2729.

[9] D. Carmier, C. Vix-Guterl, and J. Lahaye, Carbon, 2001, 39(14), 2181-2186.

[10] S.B. Lee, S.I. Pyun, and E.J. Lee, Electrochim. Acta, 2001, 47(6), 855-864.

[11] C.H. Kim and S.I. Pyun, J. Electrochem. Soc., 2003, 150(9), A1176.

[12] Y. Gao, L Chen, M. Quan, G. Zhang, G. Zheng, and J. Zhao, J. Electroanal. Chem., 2018, 808, 8-13.

[13] K. Li, Z. Xu, X. Shen, K. Yao, J. Zhao, R. Zhang, J. Zhang, L. Wang, and J. Zhu, Electrochim. Acta, 2019, 295, 569-576.

[14] C. Du, S. Liu, W. Zhang, X. Zhang, and Y. Deng, J. Electrochem. Soc., 2018, 165(9), A1955.

[15] K. Fujisawa, H.J. Kim, S.H. Go, H. Muramatsu, T. Hayasi, M. Endo, T.C. Hirschmann, M.S. Dresselhaus, Y.A. Kim, and P.T. Araujo, Appl. Sci., 2016, 6(4), 109.

[16] W.-S. Zhao, K. Fu, D.-W. Wang, M. Li, G. Wang, and W.-Y. Yin, Appl. Sci., 2019, 9(11), 2174.

[17] H. Lyu, C.J. Jafta, I. Popovs, H.M. Meyer, J.A. Hachtel, J. Huang, B.G. Sumpter, S. Dai, and X.-G. Sun, J. Mater. Chem. A, 2019, 7(30), 17888-17895.

[18] S. Qiu, G. Lu, J. Liu, H. Lyu, C. Hu, B. Li, X. Yan, J. Guo, and Z. Guo, RSC Adv, 2015, 5(106), 87286-87294.

[19] X. Li, H Gu., J. Liu, H. Wei, S. Qiu, Y. Fu, H. Lv, G. Lu, Y. Wang, and Z. Guo, RSC Adv., 2015, 5(10), 7237-7244.

[20] J. Candy, P. Fouilloux, M. Keddam, and H. Takenouti, Electrochim. Acta, 1981, 26(8), 1029-1034.

[21] F. Walsh, M. Pozin, A. Cherniy, and Jr.K. Tikhonov, $J$. Power Sources, 2001, 97, 714-718.

[22] T. Osaka, D. Mukoyama, and H. Nara, J. Electrochem. Soc., 2015, 162(14), A2529.

[23] Y. Lee, D. Kim, S. Kim, M. Kim, H. Choe, K. Lee, J. Sohn, S. Cha, J. Kim, and K. Park, ACS Appl. Mater. Interfaces, 2016, 8(11), 7022-7029.

[24] M. A. Zabara, C.B. Uzundal, and B. Ulgut, $J$. Electrochem. Soc., 2019, 166(6), A811. 